\title{
Plan de mejora enfocado en la seguridad industrial para la empresa Inmeplast basado en las $5 \mathrm{~S}$
}

\author{
Plan of progress focused in the industrial safety for the company inmeplast based $5 S$
}

Kleber Antonio Luna Altamirano. ${ }^{1}$, Laura Geordania Quizhpe Peralta. ${ }^{2}$ \& Karina Magaly Bravo Chimbo. $^{3}$

Recibido: 12-11-2019/Revisado: 02-12-209/Aceptado: 23-12-2019/ Publicado: 04-01-2020

\begin{abstract}
DOI: https://doi.org/10.33262/cienciadigital.v4i1.1074

The company Industrial Inmeplast was created on July 1st 1994, is located in the city of Cuenca-Ecuador, is dedicated to the manufacture of plastic parts and pieces in reduced times, through the use of injection machines. The problem with the research is that Inmeplast employees do not use the safety equipment constantly during their working day, causing accidents at work despite the fact that the company provides the necessary equipment (helmets, gloves, shoes, masks, goggles, earmuffs, work clothes) and even provides the appropriate training, talks or conferences to deal with the different emergencies that may arise. The objective of the study is to design an improvement plan in the production area of the company, implementing the 5S (They are the key tool to achieve the organization of the work area and each concept has an important function. Seiri: selection or classification, distinguishing what is necessary from what is not Seiton: Order or organization, a place for everything, and everything in its place. Seiso: Cleaning, establishing methods to keep the workplace clean. Seiketsu: Standardized cleaning, is the condition or state that exists when the first 3 \&quot; S"; are maintained. Shitsuke: Discipline, establish mechanisms to establish a habit). The research methodology is of a descriptive nature with a quantitative approach. The technique of surveys will be applied to obtain information that will serve as the basis for developing an improvement plan, which is a set of change measures taken in an organization with the aim of improving the production area with respect to the use of safety implements. Finally, the results will be workers committed to their safety by providing a comfortable working environment and thus become the ideal ally for the satisfaction of the organization.
\end{abstract}

\footnotetext{
${ }^{1}$ Doctor (c) en Ciencias Sociales Mención Gerencia Universidad del Zulia República Bolivariana de Venezuela, Profesor Investigador Unidad Académica de Administración Universidad Católica de Cuenca, klunaa@ucacue.edu.ec

${ }^{2}$ Estudiante de la carrera de Contabilidad y Auditoría en la Unidad Académica de Administración de la Universidad católica de Cuenca, laura.quizhpe.79@est.ucacue.edu.ec

${ }^{3}$ Estudiante de la carrera de Contabilidad y Auditoría en la Unidad Académica de Administración de la Universidad católica de Cuenca, karina.bravo.23@est.ucacue.edu.ec
} 
Keywords: Industrial safety, improvement plan, 5S, life insurance.

\section{Resumen}

La empresa Industrial Inmeplast fue creada el 01 de julio del año 1994, está ubicada en la ciudad de Cuenca-Ecuador, se dedica a la fabricación de partes y piezas plásticas en tiempos reducidos, mediante el uso de máquinas inyectoras. El problema de la investigación radica en que los empleados de la empresa Inmeplast no utilizan los implementos de seguridad constantemente en su jornada laboral, causando accidentes de trabajo a pesar de que la misma provee los implementos necesarios para laborar (cascos, guantes, zapatos, mascarillas, gafas, orejeras, ropa de trabajo) e incluso proporciona las capacitaciones adecuadas, charlas o conferencias para confrontar las diferentes emergencias que se puedan presentar. El objetivo del estudio es diseñar un plan de mejora en el área de producción de la empresa, implementando las 5S (Son la herramienta clave para lograr la organización del área de trabajo y cada concepto tiene una función importante. Seiri: selección o clasificación, distinguir lo que es necesario de lo que no lo es. Seiton: Orden u organización, un lugar para cada cosa, y cada cosa en su lugar. Seiso: Limpieza, establecer métodos para mantener limpio el lugar de trabajo. Seiketsu: Limpieza estandarizada, es la condición o el estado que existe cuando se mantienen las 3 primeras "S". Shitsuke: Disciplina, establecer mecanismos para establecer un hábito). La metodología de la investigación es de tipo descriptiva con enfoque cuantitativo, se aplicará la técnica de encuestas para obtener información que servirá de base para desarrollar un plan de mejora que es un conjunto de medidas de cambio que se toman en una organización con la finalidad de mejorar el área de producción en lo que respecta al uso de los implementos de seguridad. Finalmente, como resultados se obtendrá unos trabajadores comprometidos con su seguridad brindando un ambiente laboral confortable y así convertirse en el aliado ideal para la satisfacción de la organización.

Palabras clave: Seguridad industrial, plan de mejora, 5S, seguro de vida.

\section{Introducción}

La empresa Inmeplast fue formada en el año 1994, por el Prto. Cesar Chica Segovia, como un pequeño taller de matricería y actualmente es una empresa familiar dedicada a la elaboración de partes y piezas plásticas inyectadas de alta calidad, que permitan ser competitivos en el mercado a largo plazo, buscando la mayor participación en el mercado industrial y estando siempre a la vanguardia de la tecnología.

El problema se desprende dentro del personal de producción de la planta, ya que no utilizan los implementos de seguridad constantemente en sus jornadas laborales, causando accidentes de trabajo que perjudican el bienestar de la empresa, a pesar de que la misma provee las herramientas necesarias para laborar e incluso proporciona las capacitaciones adecuadas, charlas o conferencias para confrontar las diferentes emergencias que se puedan presentar. Y para ello se 
implementará un plan de mejora basado en el método de las 5S. Altamirano y Moreno (s.f.) afirman: "es un método practico para el establecimiento y mantenimiento del lugar de trabajo bien organizado, ordenado y limpio, a fin de mejorar las condiciones de seguridad, calidad en el trabajo y en la vida diaria" (p. 1). Nace de la filosofía japonesa, después de la segunda guerra mundial como parte de un movimiento de calidad y productividad para la recuperación económica en la industria, proviene de los términos japoneses de 5 elementos Seiri (Seleccionar: Consiste en retirar cualquier cosa que no se vaya a utilizar en los próximos 30 días), Seiton (Orden: Suministrar un lugar conveniente, seguro y ordenado a cada cosa),Seiso (Limpieza: Limpiar el entorno de trabajo incluido máquinas y herramientas), Seiketsu (Estandarización: Mantener la limpieza de la persona por medio de los equipos de protección del personal, así como mantener un entorno de trabajo saludable y limpio), Shitsuke (Autodisciplina: Logra el hábito de respetar y utilizar correctamente los procedimientos, estándares y controles previamente desarrollados)(Lagunas, 2007, p.1).

El objetivo del estudio es diseñar un plan de mejora, Francesc et al. (2005) afirman "es la propuesta de actuaciones, resultante de un proceso previo de diagnóstico de una unidad, que recoge y formaliza los objetivos de mejora y las correspondientes actuaciones dirigidas a fortalecer los puntos fuertes y resolver los débiles, de manera priorizada y temporalizada" (p.14). En el área de producción de la empresa, implementando las $5 \mathrm{~S}$ y a su vez conceder el $100 \%$ de un seguro de vida temporal que permita al empleado acceder a este en caso de muerte o accidente dentro de las horas laborables del trabajo, en caso de que no cumplan con estas normas se multara con el $30 \%$ de un salario básico, esto definirá una cultura de mejoramiento continuo, que adoptara herramientas que favorecerán el compromiso total del personal con su puesto y materiales de trabajo, para conseguir lo propuesto e inducir un cambio en sus aptitudes y actitudes de esta manera garantizar el éxito. Es decir, las 5S proponen también eliminar materiales innecesarios, mantener el orden, eliminar las fuentes de suciedad, tener un control continuo de los materiales y equipos que se usan en las diferentes áreas.

La investigación es de tipo descriptivo, Rojas (2015) asevera "Exhibe el conocimiento de la realidad tal como se presenta en una situación de espacio y de tiempo dado. Aquí se observa y se registra, o se pregunta y se registra" (p.7). En donde se busca recoger información de manera independiente con un enfoque cuantitativo Hernández (2014) afirma "Utiliza la recolección de datos para probar hipo tesis con base en la medición numérica y el análisis estadístico, con el fin establecer pautas de comportamiento y probar teorías", (p.37). La información se indica a través de la recolección de datos de manera numérica aplicando la técnica de encuestas para obtener información que servirá de base para desarrollar el plan de mejora.

Como resultados se puede visualizar un antes y un después de la implementación de las 5S tomando en cuenta los aspectos de organización, aplicación, orden y limpieza del programa de mejora, creando un ambiente laboral saludable, eficaz, con menos accidentes de trabajo y así cada trabajador estará comprometido con la empresa generando un producto de calidad. 
El artículo se estructura como primera parte con el estado del arte, en el cual se sustenta teóricamente con la aportación de otros autores que hagan mención sobre el tema a continuación se detalla la metodología de la investigación en donde se redacta los resultados de las personas que fueron encuestadas, posteriormente se muestra la matriz FODA de la empresa con un análisis externo e interno de los puntos que le favorecen y debilitan a la empresa con su respectivo plan de mejora que es un propuesta para la solución del problema implantado, y por ultimo como conclusión se lograra tener personas responsables y enfocadas en su trabajo cumpliendo con la mejora continua.

\section{Estado del arte}

Crear un plan de mejora enfocado en las 5S para la empresa Inmeplast de la ciudad CuencaEcuador, tiene como propósito corregir el estado de la empresa en el área de producción, para ello algunos autores contribuyen con sus investigación entre ellos: Cardona y Serrano (2012) proponen diseñar una plan basado en la técnica de las $5 \mathrm{~s}$ como herramienta básica para mejorar la productividad, en el que se vea reflejado primeramente el orden y el mejoramiento de cada uno de los procedimientos y niveles de calidad. Además, del compromiso no sólo del personal sino también de la alta gerencia planeando, organizando y ejecutando con cuidado cada paso a seguir para lograr mayor productividad, un lugar de trabajo con condiciones adecuadas y una mejora continua. Guachisaca y Salazar (2009) señala que con la implementación de las 5S es necesario fomentar el trabajo en equipo para que todos los trabajadores se involucren en el proceso de mejora partiendo del conocimiento de cada uno de los puestos de trabajo. Con capacitaciones harán que cada trabajador se comprometa con la empresa logrando que la mejora continua se convierta en un trabajo para todos.

Por otra parte Benavides y Castro (2010) realizan un análisis de las 5S señalan que es una técnica que promueve la mejora continua de las empresas mediante la utilización de planes de acciones correctivas ante problemas originados en las mismas, además de ello Arguello y Soto (2014) proponen realizar un plan en donde se dé a conocer los principales riesgos que se pueden presentar en la planta y cuáles serían las formas de mitigarlos donde se interactúe con los operarios y supervisores los cuales aporten sus ideas y formas de prevenir los accidentes e incidentes, también en ellas felicitara por buenas prácticas , manejo de las herramientas, elementos de protección personal y por último se da a conocer los accidentes o acciones inseguras que se generaron en las instalaciones o plantas de la empresa, para que con esto se maneje de manera total la seguridad industrial en todas las plantas y se unifiquen criterios.

Sandoval y Price (s.f.) por otra parte proponen la implementación de un programa de mejora con la finalidad de resolver los puntos más críticos, ante esta situación procediendo a formar un equipo de trabajo el cual será responsable de que esta implementación se lleve a cabo de la mejor manera siguiendo los puntos detallados según su criterio en base a la experiencia ganada en el tiempo de labores en la compañía.

Los autores mencionados, con sus contribuciones aportan al diseño del plan de mejora, ya que tienen una directa relación con la propuesta planteada, este mejorará el ambiente laboral y con 
ello tratar de obtener mejores resultados en la calidad de los productos, contribuyendo al crecimiento de la empresa.

\section{Metodología}

La presente investigación realizada es de tipo descriptivo con enfoque cuantitativo, para la elaboración de un plan de mejora en seguridad industrial, la información será proporcionada a través de encuestas dirigidas a 25 empleados del área de producción de la empresa INMEPLAST en la ciudad de Cuenca-Ecuador, con el afán de recolectar información veraz.

Tabla 1. Segmentación de empleados de la empresa Inmeplast

\begin{tabular}{lc}
\hline \multicolumn{1}{c}{ Personal Total } & Número \\
\hline Jefe de planta & 1 \\
Montajista & 1 \\
Operadores de Maquina & 23 \\
\hline \multicolumn{1}{c}{ Total, de empleados } & $\mathbf{2 5}$ \\
\hline
\end{tabular}

Fuente: Elaboración propia

\section{Resultados}

En la tabla 1, elaborada con anterioridad se muestra que la población de los trabajadores que conforman la empresa Inmeplast de la ciudad de Cuenca-Ecuador del área de producción son 25 empleados encuestados, teniendo en cuenta que sus edades varían entre los 20 hasta 50 años, considerando que cada empleado fue capacitado para ocupar el cargo en el que se desempeñan y están conscientes de la problemática de la empresa, la información recopilada de las encuestas se presenta a continuación.

Figura 1. Plan de Mejora

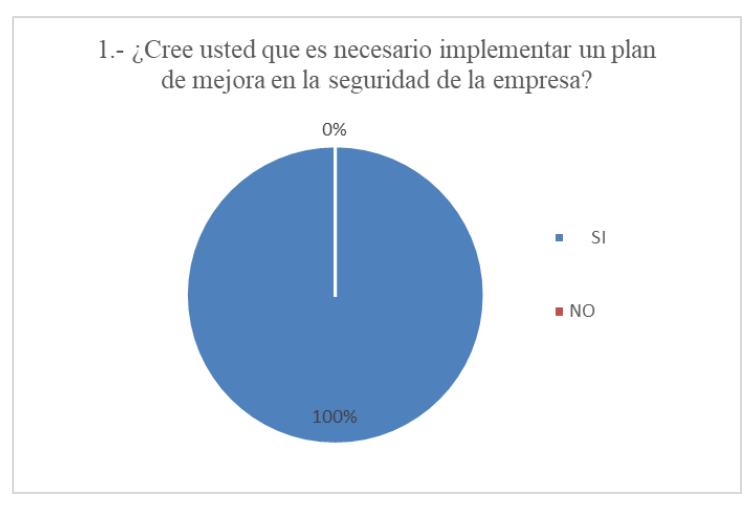

Fuente: Elaboración propia

Se puede observar en el gráfico número 1, que en la empresa Inmeplast consideran que si es necesario tener un plan de mejora. Cárdenas y Fecci (2007) afirman:

El mejoramiento continuo es una herramienta fundamental para todas las empresas porque les permite renovar los procesos administrativos que ellos realizan, lo cual 
hace que las organizaciones estén en constante actualización; además permite que sean más eficientes y competitivas, fortalezas que le ayudarán a permanecer en el mercado (p. 4)

Figura 2. 5S

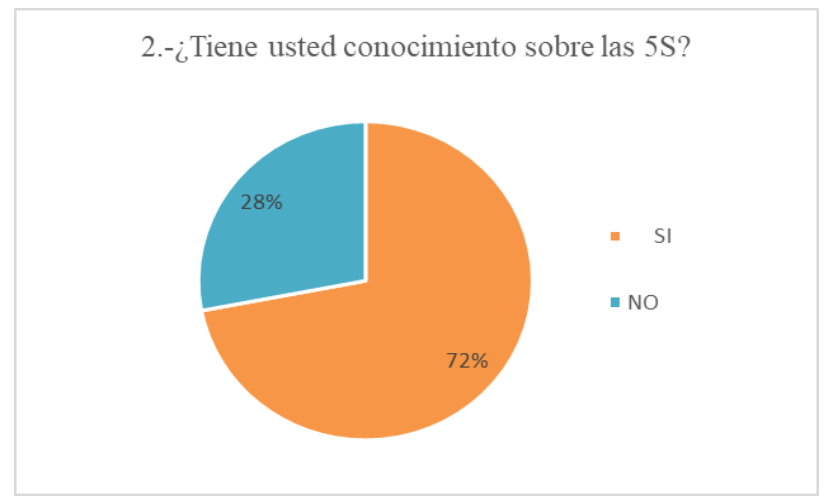

Fuente: Elaboración propia

Se puede observar en el gráfico número 2, que en la empresa Inmeplast el $28 \%$ de las personas encuestada si tiene conocimiento sobre las $5 \mathrm{~S}$ y el $72 \%$ restante no.

La 5S puede representarse como un sistema que posibilita la creación de las condiciones necesarias para la implementación de nuevas soluciones técnicas; se basa en ideas innovadoras, la optimización del espacio de trabajo y el proceso de producción se realizan también; adopta un enfoque sistemático que implica el trabajo en equipo, incluyendo la participación de todos los empleados, y se centra en la aplicación total de la organización y la adaptación del espacio de trabajo (Vorkapić, Coćkalo, Dordević y Besić, 2017).

Figura 3 Implementos

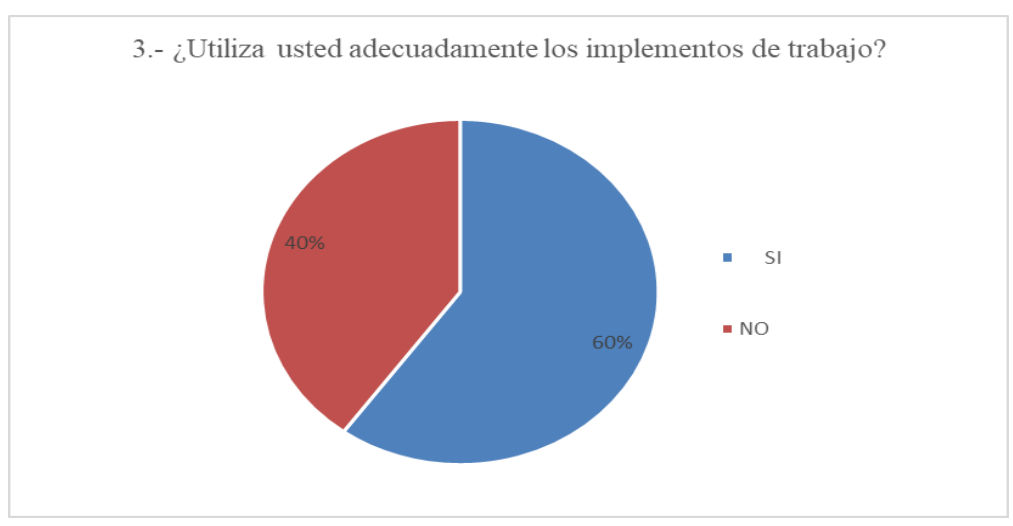

Fuente: Elaboración propia

Se puede observar en el gráfico número 3, que en la empresa Inmeplast el 40\% de los empleados no utilizan adecuadamente los implementos necesarios de seguridad. Y el 60\% sí. 
Universidad del valle (2006) afirma "cualquier equipo o dispositivo destinado a ser utilizado por el trabajador, para protegerlo de uno o varios riesgos y aumentar su seguridad o su salud en el trabajo" (p. 4)

Figura 4. Control implementos de trabajo

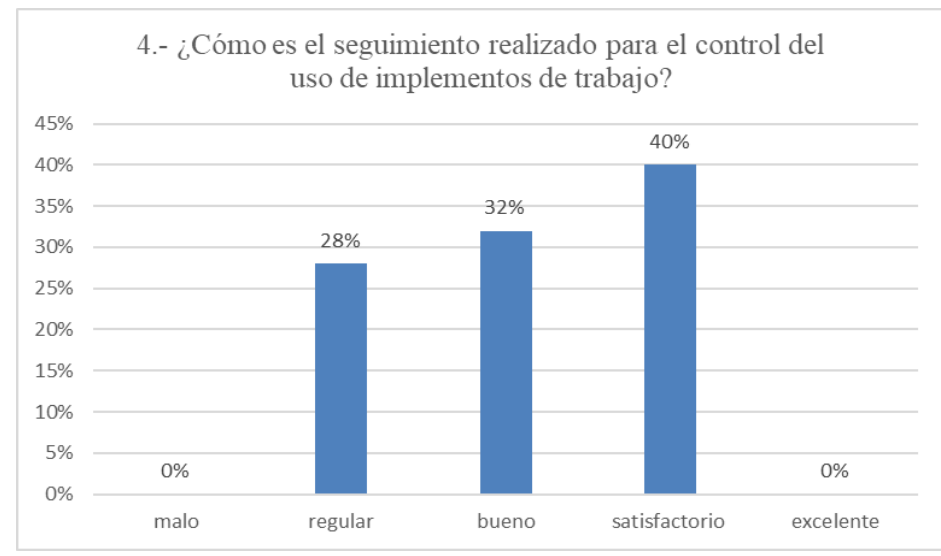

Fuente: Elaboración propia

Se puede observar en el gráfico número 4, que en la empresa Inmeplast 10 personas que representan el $40 \%$ del personal de producción indican que el manejo del control de uso de los implementos es satisfactorio, seguido del $32 \%$ y del $28 \%$, que mencionan que dicho seguimiento es bueno y regular respectivamente.

CMIC (2012), la supervisión es una actividad técnica y especializada que tiene como fin fundamental utilizar racionalmente los factores que le hacen posible la realización de los procesos de trabajo: el hombre, la materia prima, los equipos, maquinarias, herramientas, dinero, entre otros elementos que en forma directa o indirecta intervienen en la consecución de bienes, servicios y productos destinados a la satisfacción de necesidades de un mercado de consumidores, cada día más exigente, y que mediante su gestión puede contribuir al éxito de la empresa (p.1).

Figura 5 Organización de materiales

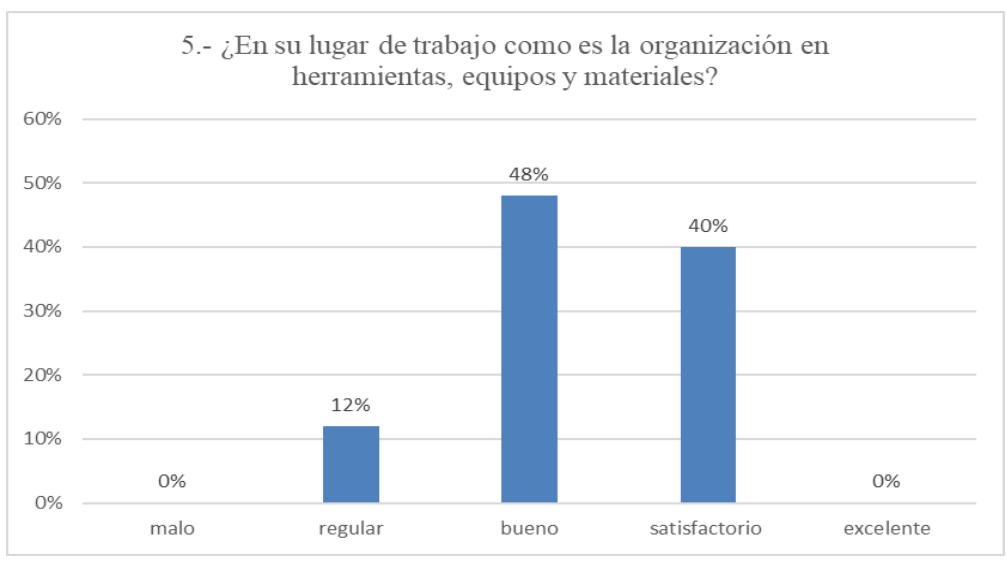

Fuente: Elaboración propia 
El manejo bueno de herramientas, equipos y materiales representa el $48 \%$, seguido del $40 \%$ y $12 \%$ que emplean de forma satisfactoria y regular respectivamente.

Es un programa de trabajo que consiste en desarrollar actividades de orden/limpieza y detección de anomalías en el puesto de trabajo, que por su sencillez permiten la participación de todos a nivel individual/grupal, mejorando el ambiente de trabajo, la seguridad de personas y equipos, y la productividad (Rey, 2005).

Figura 6 Estandarización

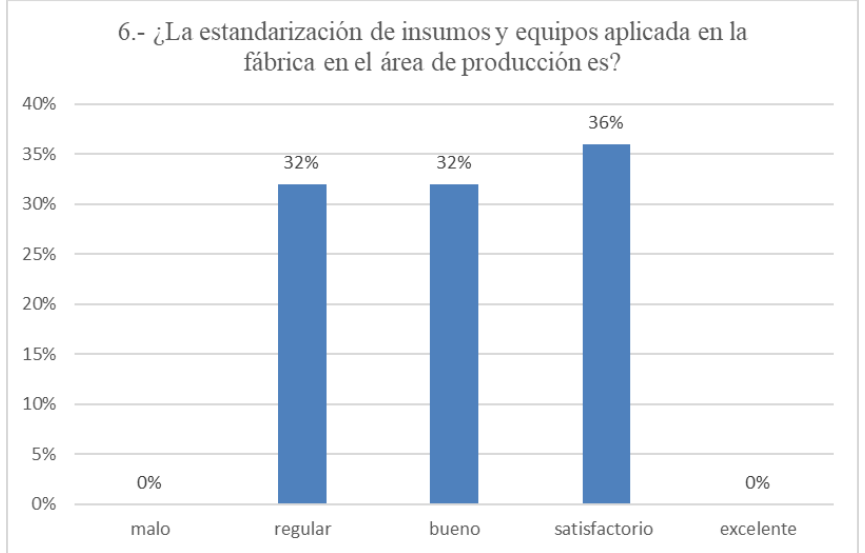

Fuente: Elaboración propia

Inmeplast adecuada de forma satisfactoria con un $36 \%$ la estandarización de insumos y equipos, seguido de un manejo bueno y regular en un $32 \%$ en ambas categorías.

Manene (2010), el primer paso de la Mejora en cualquier tipo de Empresas es el Orden y la Limpieza, Si deseamos Mejorar primero tenemos que estandarizar, imagínense un lugar en donde no podamos encontrar lo que buscamos, que el exceso de material terminado y materia prima nos impida ver las áreas de oportunidad, es hay en donde el Programa de las 5S inicial, la relación trabajador-gerencia y que permita que todo el personal participe activamente del proceso de mejora continua.

Figura 7 Limpieza

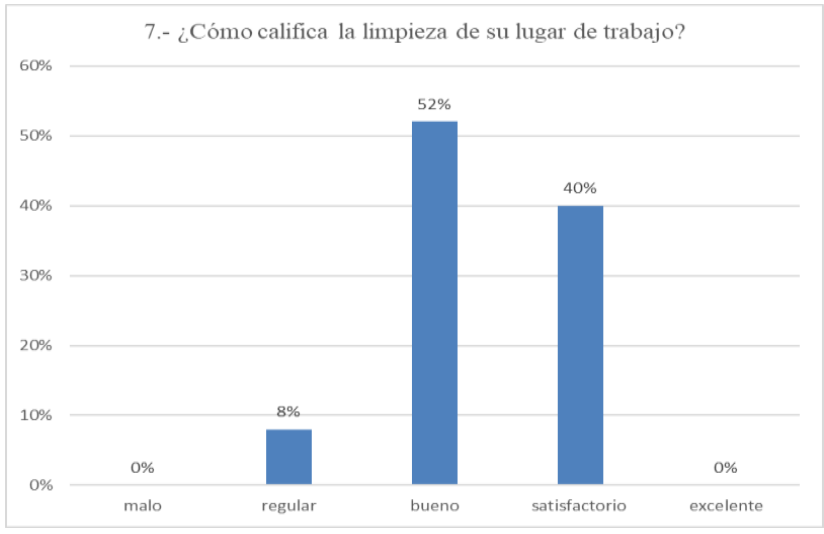

Fuente: Elaboración propia 
Los empleados califican que la limpieza en la fábrica se da de forma buena representado con un $52 \%$, estos también insinúan que la limpieza en la empresa es satisfactoria representado por el $40 \%$, y tan solo el $8 \%$ indican que esto de ejecuta de forma regular.

5S es la metodología de creación y mantenimiento de un lugar de trabajo bien organizado, limpio, de alta eficacia y de alta calidad (Shaikh, et al, 2015).

Figura 8 Disciplina

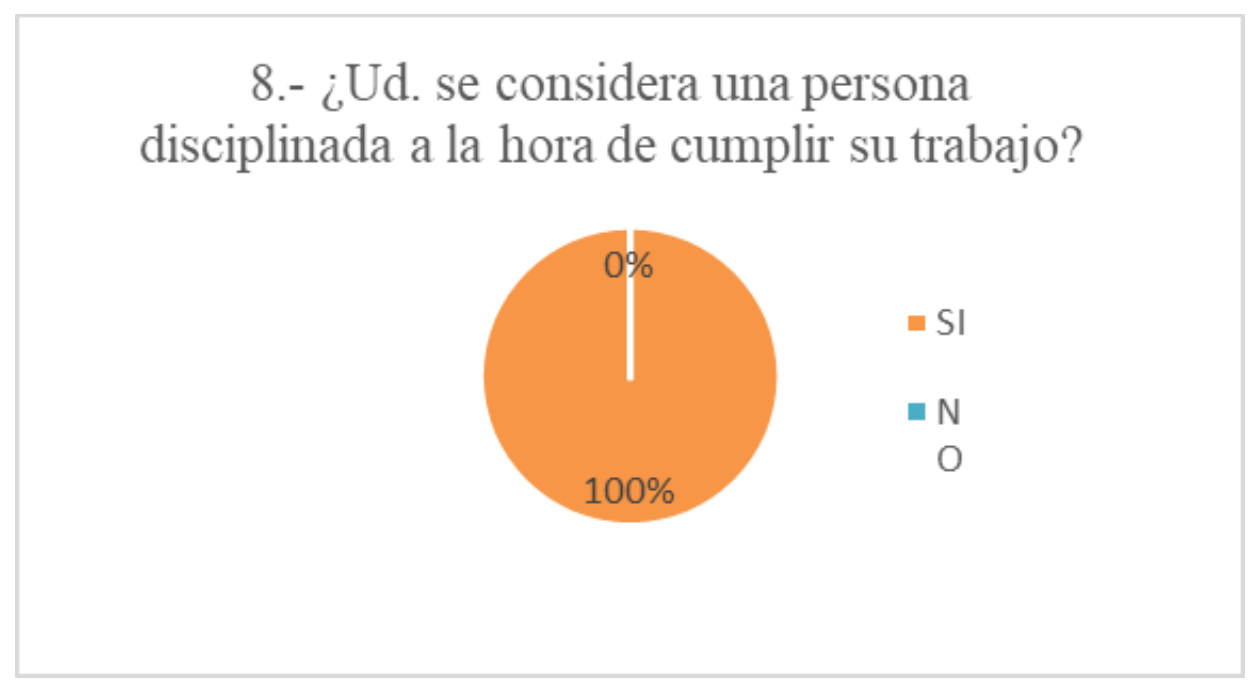

Fuente: Elaboración propia

Los empleados indican que realizan sus actividades de forma disciplinada representado por el $100 \%$, como disciplina práctica, se asume como aquella encargada del conjunto de procesos de planeación, organización, dirección y control en las organizaciones. Martínez (2013) explica como incentivo para el empleado que cumpla con el plan de mejora se otorgara el 100\% de un seguro de vida privado como consecuencia de no cumplir con las normas planteadas se multara con el 30\% del salario básico unificado, esto permitirá concientizar, y crear responsabilidad o cierto tipo de obligación al personal en sus diferentes turnos de trabajo, de esta manera se prepara para cualquier tipo de situación emergente, es decir no depende de los recursos materiales sino de la participación y compromiso de los trabajadores reduciendo así el problema.

Para el diseño del modelo de un plan de mejora de la empresa Inmeplast, se estructuró un análisis FODA el cual consiste en realizar una evaluación de los factores fuertes y débiles de la empresa es decir la situación interna, y sus oportunidades y amenazas como factores externos, esto permitirá visualizar la situación en la que se encuentra a continuación se refleja en la siguiente tabla: 
Tabla 2. Matriz FODA Factores Internos y Externos

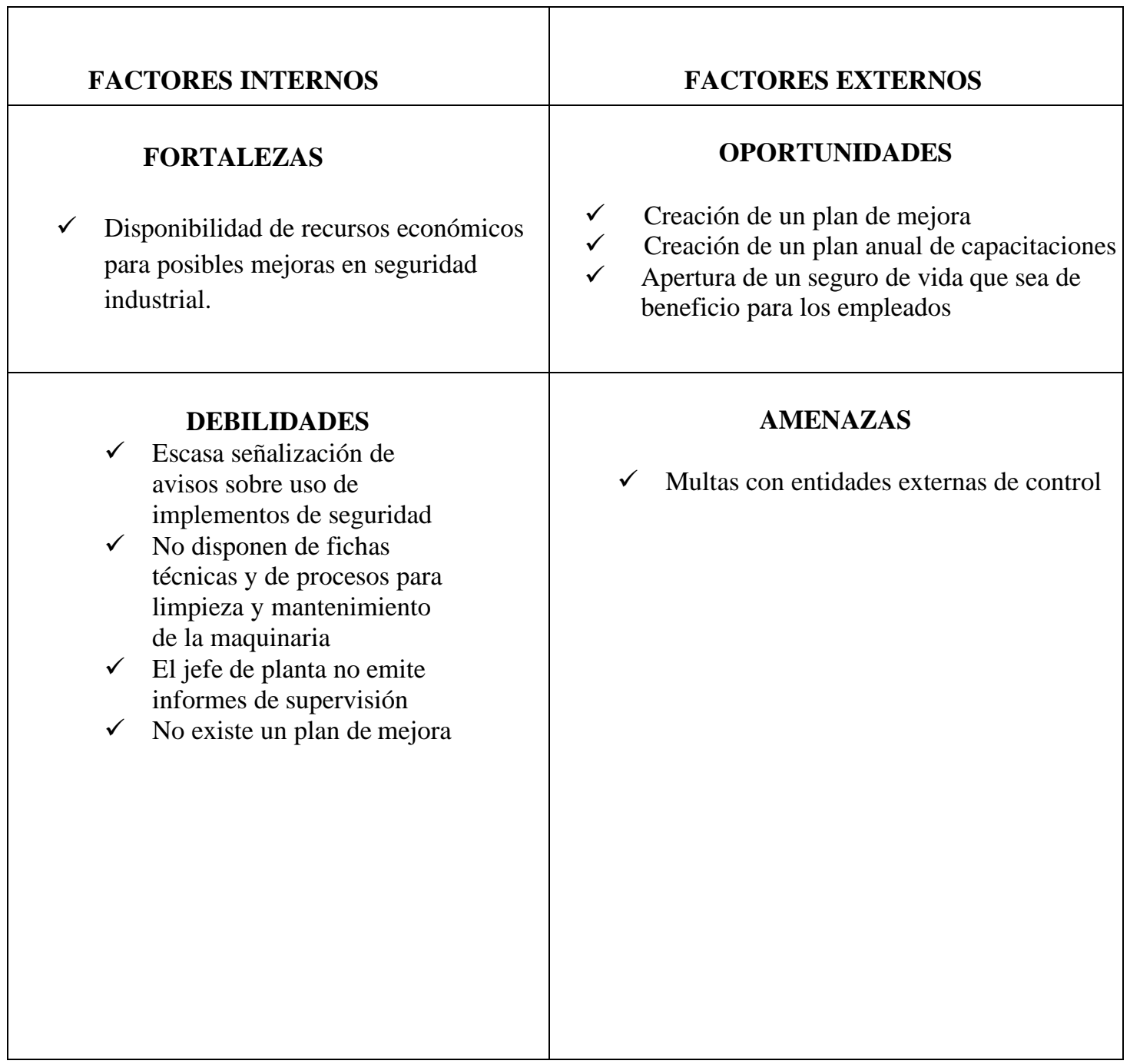

Fuente: Elaboración propia

\section{Plan de mejora}

Partiendo de la tabla 2, se diseña un plan de mejora enfocado en las 5S para corregir los problemas en cuanto a seguridad industrial, como primer paso es separar, es decir lo útil de lo inútil para el desenvolvimiento normal de las actividades, como segundo paso ordenar es decir que en cada área el colaborador organizara sus implementos de manera que pueda verlos y acceder a ellos fácilmente, como tercer paso limpiar es decir garantizar que los elementos se encuentren limpios y listos para ser utilizados cuando se requiera, el cuarto paso estandarizar en este paso se crea procedimientos para utilizar los implementos y materiales, y como quinto y último paso la autodisciplina, siendo el más importante ya que permite verificar el seguimiento de los pasos anteriores para así tomar medidas correctivas. 


\section{Tabla 2 Plan de Mejora}

\begin{tabular}{|c|c|c|c|c|c|}
\hline $\begin{array}{l}\text { Problema } \\
\text { planteado }\end{array}$ & Acciones propuestas & Resultados & 5 "S" & Responsable & \begin{tabular}{|l|} 
Tiempo \\
estimado
\end{tabular} \\
\hline $\begin{array}{l}\text { Los empleados } \\
\text { no solicitan los } \\
\text { implementos } \\
\text { de seguridad } \\
\text { necesarios al } \\
\text { inicio del turno }\end{array}$ & $\begin{array}{l}\text { Crear una hoja de control } \\
\text { semanal, en donde una } \\
\text { persona encargada } \\
\text { entregará los mismos y } \\
\text { dará seguimiento a que } \\
\text { implementos se utilizan y } \\
\text { quienes cumplen con las } \\
\text { normas }\end{array}$ & $\begin{array}{l}\text { Como resultado } \\
\text { se obtendrá un } \\
\text { mejor control de } \\
\text { los implementos } \\
\text { que se usan con } \\
\text { frecuencia }\end{array}$ & $\begin{array}{l}\text { SELECCIONAR } \\
\text { SEIRI }\end{array}$ & $\begin{array}{l}\text { Jefe de } \\
\text { planta }\end{array}$ & 7 días \\
\hline $\begin{array}{l}\text { No se cuenta } \\
\text { con las } \\
\text { herramientas } \\
\text { de trabajo en su } \\
\text { lugar } \\
\text { respectivo }\end{array}$ & $\begin{array}{l}\text { Realizar una zona de } \\
\text { almacenamiento } \\
\text { alfabética de herramientas } \\
\text { en donde cada trabajador } \\
\text { pueda encontrar la misma } \\
\text { con facilidad }\end{array}$ & $\begin{array}{l}\text { Se ahorrará el } \\
\text { tiempo del } \\
\text { trabajador a la } \\
\text { hora que tenga } \\
\text { un inconveniente } \\
\text { con la máquina y } \\
\text { necesite usar una } \\
\text { herramienta } \\
\end{array}$ & $\begin{array}{l}\text { ORDENAR } \\
\text { SEITON }\end{array}$ & $\begin{array}{l}\text { Jede de } \\
\text { planta } \\
\text { /Operador de } \\
\text { maquina }\end{array}$ & 15 días \\
\hline $\begin{array}{l}\text { Identificar de } \\
\text { donde proviene } \\
\text { más la } \\
\text { suciedad }\end{array}$ & $\begin{array}{l}\text { Creación de un } \\
\text { procedimiento de } \\
\text { limpieza de cada área de } \\
\text { trabajo en donde se } \\
\text { determinará la frecuencia } \\
\text { y forma de limpieza. }\end{array}$ & $\begin{array}{l}\text { De esta manera } \\
\text { aseguraremos la } \\
\text { limpieza del área }\end{array}$ & $\begin{array}{l}\text { LIMPIAR } \\
\text { SEISO }\end{array}$ & $\begin{array}{l}\text { Operador de } \\
\text { maquina /jefe } \\
\text { de grupo }\end{array}$ & 30 días \\
\hline $\begin{array}{l}\text { Pérdida o hurto } \\
\text { constante de } \\
\text { implementos } \\
\text { de seguridad }\end{array}$ & $\begin{array}{l}\text { Implementar un lugar en } \\
\text { donde estén los } \\
\text { implementos de seguridad } \\
\text { con una ficha en la cual } \\
\text { cada empleado al obtener } \\
\text { la misma tendrá q firmar } \\
\text { de esta manera se llevará } \\
\text { un control }\end{array}$ & $\begin{array}{l}\text { Evitar } \\
\text { desperdicio de } \\
\text { implementos. }\end{array}$ & $\begin{array}{c}\text { ESTANDARIZAR } \\
\text { SEIKETSU }\end{array}$ & $\begin{array}{l}\text { Operador de } \\
\text { maquina }\end{array}$ & 90 días \\
\hline $\begin{array}{l}\text { Uso incorrecto } \\
\text { de } \\
\text { implementos } \\
\text { de seguridad }\end{array}$ & $\begin{array}{l}\text { Crear una hoja de control } \\
\text { semanal, en donde se } \\
\text { verificará diariamente si } \\
\text { los empleados usan estos } \\
\text { implementos. } \\
\\
\text { Para motivar a los } \\
\text { trabajadores a que } \\
\text { cumplan con el uso } \\
\text { debido de los } \\
\text { implementos de seguridad } \\
\text { la empresa concederá el } \\
\text { 100\% de un SEGURO } \\
\text { DE VIDA, caso contrario } \\
\text { se descontara el 30\% del } \\
\text { rol como multa }\end{array}$ & $\begin{array}{l}\text { Determinar las } \\
\text { acciones } \\
\text { correctivas para } \\
\text { cada problema. }\end{array}$ & $\begin{array}{l}\text { AUTODISCIPLINA } \\
\text { SHITSUKE } \\
\end{array}$ & $\begin{array}{l}\text { Jefe de } \\
\text { planta }\end{array}$ & 7 días \\
\hline
\end{tabular}

Fuente: Elaboración propia 


\section{Conclusiones}

El diseñar un plan de mejora para la empresa Inmeplast de la ciudad Cuenca-Ecuador basado en las 5S tiene como propósito concientizar a los empleados para el uso correcto de las herramientas de trabajo, la propuesta presentada en esta investigación cumple con el objetivo planteado y se orienta a gestionar de una mejor manera el área de producción. Con este estudio se propende a mejorar la seguridad de los trabajadores evitando accidentes laborales, e impedir que entidades externas de supervisión realicen visitas sorpresas y generen sanciones, con ello se pretende crear una cultura de seguridad y compromiso laboral con la organización.

Como beneficio para los trabajadores se otorgará y se cubrirá un seguro de vida, como incentivo a la utilización de herramientas de seguridad. Con el apoyo de este método de las 5S la empresa, entra en un proceso de mejora continua a través de un mayor rendimiento de sus procesos, con ello se trata de reducir el costo del riesgo y el impacto de un accidente que podría ocurrir dentro del entorno productivo.

Este estudio aporta a un mayor rendimiento en el trabajo por parte de sus obreros, reduciendo los accidentes, mayor eficacia y prevención de cualquier incidente. La fidelidad de sus obreros a la empresa se incrementará a través del mejoramiento de su calidad de vida, la motivación hacia un mejor trabajo es consecuencia directa de una prevención de alto nivel.

Con este plan de mejora, se logrará una mayor imagen de la organización, desde una óptica interna y externa, por medio de la prevención, alineándose a una empresa competitiva, segura y eficiente, ya que esta se encaminará a fomentar un ambiente de trabajo satisfactorio y con ello obtener un mejor posicionamiento en el mercado a través de un resultado optimo y diferenciador.

\section{Referencias Bibliográficas}

Altamirano, R., y Moreno, M. (2013). Aplicación de la metodología japonesa de calidad 5 s para optimizar las operaciones en el laboratorio de mecánica de patio de la universidad de las fuerzas armadas-espe. Recuperado de: https://repositorio.espe.edu.ec/bitstream/21000/7335/1/AC-ESPEL-MAI-0443.pdf

Arguello, J., y Soto, A. (2014). Diseño e implementación del plan de mejoramiento de las condiciones de higiene y seguridad industrial en armalco s. a (fontibón siberia) (Tesis Tecnológica). Universidad distrital Francisco José de caldas, Bogotá, Colombia.

Benavides, K., Castro, P. (2010). Diseño e implementación de un programa de 5s en industrias metalmecánicas san judas Ltda. (Tesis de grado). Universidad de Cartagena, Cartagena, Colombia.

Cardona, G., y Serrano, L. (2012). Propuesta guía basada en la técnica de las 5s como herramienta básica para mejorar la productividad en la bodega de la unidad regional semap (Tesis de grado). Universidad del valle sede pacífico, Buenaventura, Colombia. 
Cárdenas, L., Fecci., E. (2007). Propuesta de un modelo de gestión para PYMEs, centrado en la mejora continua. Síntesis Tecnológica, 3 (2), 59-67. Recuperado de: http://revistas.uach.cl/pdf/sintec/v3n2/art02.pdf

CMIC, V. (2012). La supervisión y la organización. Recuperado de http://www.cmicvictoria.org/wp-content/uploads/2012/06/La-supervision-y-control-en-laAdmnistracion.pdf

Francesc, P., Francesc, A., Esteve, A., Meritxell, C., Gimeno, S., Gomez, J... Vilardell, I. (2005). Marco general para el establecimiento, el seguimiento y la revisión de los planes de mejora. Agencia per a la Qualitat del sistema universitario de la Catalunya. Recuperado de: http://www.aqu.cat/doc/doc_40159984_1.pdf

Guachisaca, C., y Salazar M. (2009). Implementación de 5S como una metodología de mejora en una Empresa de Elaboración de Pinturas (tesis de posgrado). Escuela superior politécnica de litoral, Guayaquil, Ecuador.

Hernández, R., Fernández, C., y Baptista, L. (2014). Metodología de la investigación. México: McGRAW-HILL. Recuperado de: http://observatorio.epacartagena.gov.co/wpcontent/uploads/2017/08/metodología-de-la-investigacion-sexta-edicion.compressed.pdf

Kaushik, P.; Khatak, N. y Kaloniya, J. (2015). Analyzing relevance and performance of 5S methodology: a review. International Journal of Advanced Research in Engineering and Applied Sciences, 4(4), 21-33. http://www.garph.co.uk/IJAREAS/Apr2015/3.pdf

Lagunas, J. (2007). Generalidades de las 5 "S". http://tesis.uson.mx/digital/tesis/docs/17812/Capitulo2.pdf

Manene, L., (2010). Las "9 S" y el plan de colaboración en el puesto de trabajo: pcpt. Recuperado de: http://www.luismiguelmanene.com/2010/11/22/las9-s-y-el-plande-colaboracion-en-el-puesto-de-trabajopcpt/

Martínez, C. (2013). La información en la disciplina administrativa. Revista e-Ciencias de la Información, 3 (2), 1-15. https://www.redalyc.org/pdf/4768/476848738004.pdf

Proaño, D., Gisbert, V., y Pérez, E. (2017). Metodología para elaborar un plan de mejora continua. 3C Empresa: investigación y pensamiento crítico, Edición Especial, 50-56. https://www.3ciencias.com/wp-content/uploads/2018/01/art_6.pdf

Rey, F. (2005). Las 5S. Orden y limpieza en el puesto de trabajo en España. Recuperado de https://books.google.com.ec/books?id=NJtWepnesqAC \& printsec=frontcover\&dq=inauth r:\%22Francisco+Rey+Sacrist\%C3\%A1n\%22\&hl=es419\&sa=X\&ved=0ahUKEwip1Ouy5 bmAhUMWs0KHQ4gCPoQ6wEIMDAB\# $\mathrm{v}=$ onepage $\& \mathrm{q} \& \mathrm{f}=$ false

Rojas, M. (2015). Tipos de Investigación científica: Una simplificación de la complicada incoherente nomenclatura y clasificación. REDVET. Revista Electrónica de Veterinaria 16(1), 1-14. Recuperado de: https://www.redalyc.org/pdf/636/63638739004.pdf 
Shaikh, S.; Alam, A.; Ahmed, K.; Ishtiyak, S. \& Hasan, S. (2015). Review of 5S

Technique.International Journal of Science, Engineering and Technology

Research, 4(4), 927-931. Recuperado de: http://ijsetr.org/wpcontent/uploads/2015/04/IJSETR-VOL-4-ISSUE-4-927-931.pdf

Sandoval, A., y Price M. (s.f.). Diseño de plan de mejora continua aplicando la metodología PHVA en la empresa de Emulsiones \& Asfaltos SAC. https://www.usmp.edu.pe/PFII/pdf/20142_7.pdf

Santoyo, F., Murguía, D., López, A., y Santoyo, E. (2013). Comportamiento y organización. Implementación del sistema de gestión de la calidad de 5S'S. Diversitas: Perspectivas en Psicología, 9(2), 361-371. https://www.redalyc.org/pdf/679/67932397009.pdf

Universidad del Valle. (2006). Cartilla de Elementos de Protección Personal. Recuperado de: http://saludocupacional.univalle.edu.co/CartillaEpp. pdf

Venegas, Jenny (2010). Plan para la implementación de un sistema de seguridad y salud ocupacional en la empresa Embomachala S.A. (Tesis maestría). Universidad Politécnica Salesiana, Cuenca, Ecuador.

Vorkapić, M., Coćkalo, D., Dordević, D. y Besić, C. (2017). Implementation of 5s tools as a starting point in business process reengineering. Journal of Engineering Management and Competitiveness, 7(1), 44-54. https://scindeks-clanci.ceon.rs/data/pdf/23349638/2017/2334-96381701044V.pdf

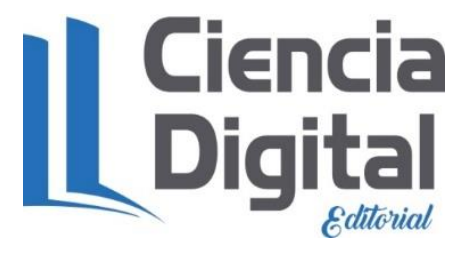


PARA CITAR EL ARTÍCULO INDEXADO.

Luna Altamirano, K. A., Quizhpe Peralta, L. G., \& Bravo Chimbo, K. M. B. C. (2020). Plan de mejora enfocado en la seguridad industrial para la empresa Inmeplast basado en las 5S. Ciencia Digital, 4(1), 111-125. https://doi.org/10.33262/cienciadigital.v4i1.1074

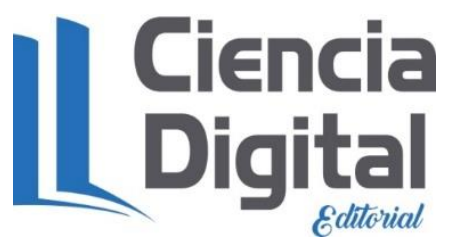

El artículo que se publica es de exclusiva responsabilidad de los autores y no necesariamente reflejan el pensamiento de la Revista Ciencia Digital.

El artículo queda en propiedad de la revista y, por tanto, su publicación parcial y/o total en otro medio tiene que ser autorizado por el director de la Revista Ciencia Digital.
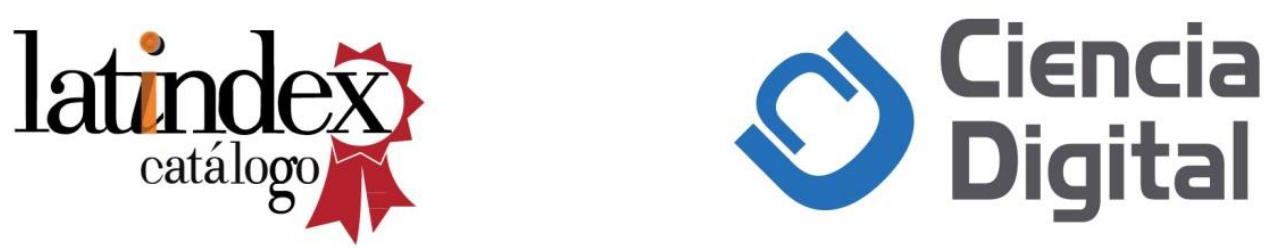